PROCEEDINGS OF THE

AMERICAN MATHEMATICAL SOCIETY

Volume 139, Number 7, July 2011, Pages 2605-2614

S 0002-9939(2010)10800-6

Article electronically published on December 23, 2010

\title{
MINIMAL SEQUENCES OF REIDEMEISTER MOVES ON DIAGRAMS OF TORUS KNOTS
}

\author{
CHUICHIRO HAYASHI AND MIWA HAYASHI
}

(Communicated by Daniel Ruberman)

Dedicated to Professor Akio Kawauchi for his 60th birthday

\begin{abstract}
Let $D(p, q)$ be the usual knot diagram of the $(p, q)$-torus knot; that is, $D(p, q)$ is the closure of the $p$-braid $\left(\sigma_{1}^{-1} \sigma_{2}^{-1} \cdots \sigma_{p-1}^{-1}\right)^{q}$. As is well-known, $D(p, q)$ and $D(q, p)$ represent the same knot. It is shown that $D(n+1, n)$ can be deformed to $D(n, n+1)$ by a sequence of $\{(n-1) n(2 n-1) / 6\}+1$ Reidemeister moves, which consists of a single RI move and $(n-1) n(2 n-1) / 6$ RIII moves. Using cowrithe, we show that this sequence is minimal over all sequences which bring $D(n+1, n)$ to $D(n, n+1)$.
\end{abstract}

\section{INTRODUCTION}

A Reidemeister move is a local move of a link diagram in the 2-sphere or the plane $\mathbb{R}^{2}$ as in Figure 1. An RI (resp. II) move creates or deletes a monogon face (resp. a bigon face). An RIII move is performed on a 3-gon face, deleting it and creating a new one. Any such move does not change the link type. As Alexander and Briggs [2] and Reidemeister [10] showed, for any pair of diagrams $D_{1}, D_{2}$ which represent the same link type, there is a finite sequence of Reidemeister moves which deforms $D_{1}$ to $D_{2}$.
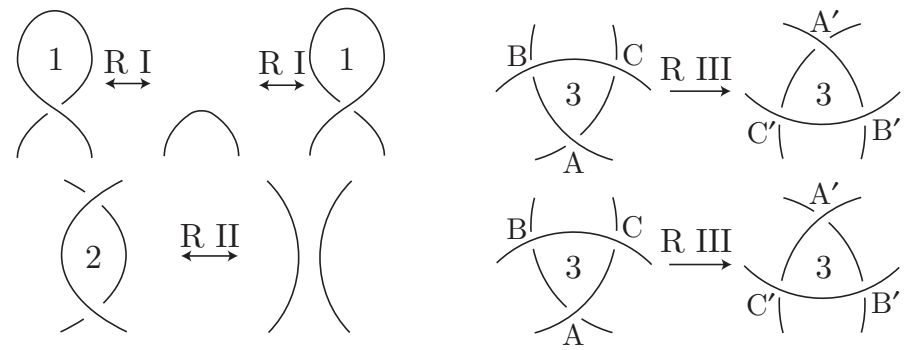

FiguRE 1. Reidemeister moves

Received by the editors March 10, 2010 and, in revised form, June 19, 2010.

2010 Mathematics Subject Classification. Primary 57M25.

Key words and phrases. Knot diagram, Reidemeister move, cowrithe, torus knot, positive knot.

The first author is partially supported by Grant-in-Aid for Scientific Research (No. 18540100), Ministry of Education, Science, Sports and Technology, Japan.

(C)2010 American Mathematical Society Reverts to public domain 28 years from publication 
Arnold introduced three invariants $S t, J^{+}$and $J^{-}$of generic planar closed curves in [1. St remains unchanged by a homotopy going through a curve with a selftangency point, but changes by one by a homotopy going through a curve with a triple point. $J^{+}$(resp. $J^{-}$) remains unchanged by a homotopy going through a curve with a triple point or an inverse (resp. direct) self-tangency point, but changes by two by a homotopy going through a curve with a direct (resp. inverse) self-tangency point. In [5], the knot diagram invariant cowrithe is introduced. It is not changed by an RI move and is changed by zero or one by an RII move and by one by an RIII move. Hence, if two knot diagrams represent the same knot, then the difference of their cowrithes gives a lower bound for the number of RII and RIII moves required for deforming one to the other. In [3], Carter, Elhamdadi, Saito and Satoh gave a lower bound for the number of RIII moves by using extended $n$-colorings of knot diagrams in $\mathbb{R}^{2}$. Hass and Nowik introduced a certain knot diagram invariant by using smoothing and linking numbers in [6], and gave in [7] an example of an infinite sequence of diagrams of the trivial knot such that the $n$-th one has $7 n-1$ crossings, can be unknotted by $2 n^{2}+3 n$ Reidemeister moves, and needs at least $2 n^{2}+3 n-2$ Reidemeister moves for being unknotted. See also [4, 8] and [9].

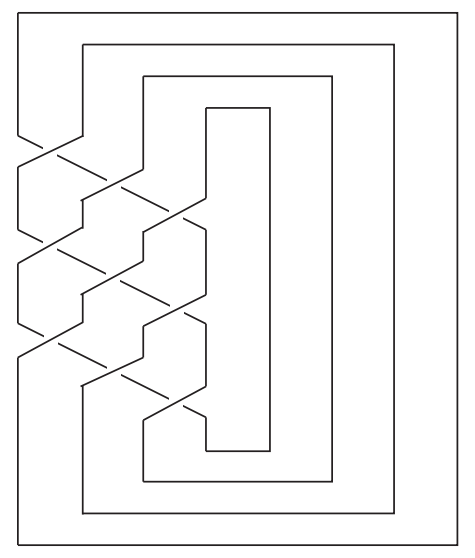

FiguRe 2. $D(4,3)$

In this paper, we study the minimal number of Reidemeister moves on diagrams of torus knots and show that the cowrithe gives exact lower bounds for $(n+1, n)$ torus knots. Let $D(p, q)$ be the usual knot diagram of the $(p, q)$-torus knot. See Figure 2, where $D(4,3)$ is depicted. To be precise, for any $i \in\{1,2, \ldots, p-1\}$, let $\sigma_{i}$ be the generator of the $p$-braid group $B_{p}$, which denotes the braid where the $i$-th strand crosses over the $(i+1)$-st strand (Figure 3). We let $b_{i}$ denote $\sigma_{i}^{-1}$ for short. Then, $D(p, q)$ is the closure of the $p$-braid $\left(b_{1} b_{2} \cdots b_{p-1}\right)^{q}$. Thus $D(p, q)$ is a positive knot diagram. In fact, it has $(p-1) q$ positive crossings and no negative crossing with respect to the usual definition of sign of a crossing (Figure 44). As is well-known, $D(p, q)$ and $D(q, p)$ represent the same knot, and hence there is a sequence of Reidemeister moves which brings $D(p, q)$ to $D(q, p)$. Using cowrithe, we obtain the following theorem. 


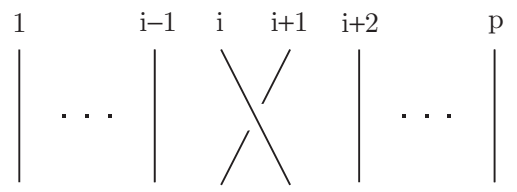

FiguRE $3 . \sigma_{i}$

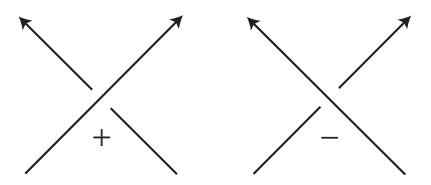

FiguRE 4. Signs of crossings

Theorem 1.1. For any integer $n$ larger than or equal to 2 , the usual positive knot diagram $D(n+1, n)$ of the $(n+1, n)$-torus knot can be deformed to $D(n, n+1)$ by a sequence of $(n-1) n(2 n-1) / 6+1$ Reidemeister moves, which consists of $(n-1) n(2 n-1) / 6$ RIII moves and a single RI move deleting a monogon face, and contains no move creating a negative crossing. Moreover, any sequence of Reidemeister moves bringing $D(n+1, n)$ to $D(n, n+1)$ must contain at least one RI move and at least $(n-1) n(2 n-1) / 6$ RII or RIII moves. Hence, the above sequence is minimal.

This theorem can also be obtained by using Arnold invariants. Hass and Nowik showed in section 4 in [6] that any lower bound of the number of Reidemeister moves between two knot diagrams obtained from the cowrithe coincides with that from the sum of adequately normalized Arnold invariants $S t+J^{+} / 2$ of the underlying spherical closed curve.

The sequence of Reidemeister moves in the above theorem is described in Section 2. The estimation for the number of RI moves is easily obtained by using writhe. The definition of cowrithe is reviewed in Section 3, where we calculate cowrithes of $D(n+1, n)$ and $D(n, n+1)$. The proof of the theorem is given in Section 4. Notes for general $D(p, q)$ and positive knots are given in Section 5 .

\section{Deformation of $D(n+1, n)$ to $D(n, n+1)$}

In this section, we deform $D(n+1, n)$ to $D(n, n+1)$ by a sequence of $(n-1) n(2 n-1) / 6+1$ Reidemeister moves. The deformation is described in Figures 5 through 12, $D(n+1, n)$ is the closed $(n+1)$-braid as in Figure 5 . We call a string of a braid the $k$-th string and denote it by $\gamma_{k}$ if it contains the $k$-th point from the left at the top of the braid. First we move the $(n+1)$-st string $\gamma_{n+1}$ going through the first $b_{n}$, the second $b_{n-1}, \cdots$ and the last $b_{1}$ of $D(n+1, n)$, so that $\gamma_{n+1}$ passes over all the crossings below it. See Figure 5, where $\gamma_{n+1}$ is drawn in the bold line and is moved to the broken line. This can be achieved by $(n-1) n / 2$ RIII moves. The first one is performed on the trigonal face marked " 3 ". Then we have a monogon face as in Figure 6, and it is eliminated by a single RI move. A closed $n$-braid is obtained as in Figure 7 . When $n=2$, we have obtained $D(2,3)$. 


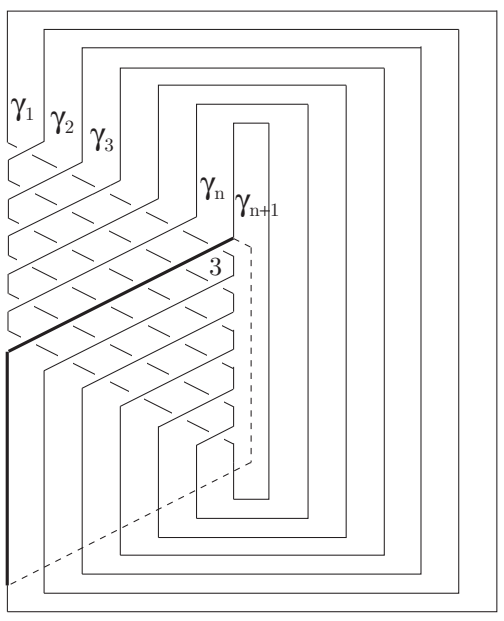

Figure 5. $D(7,6)$

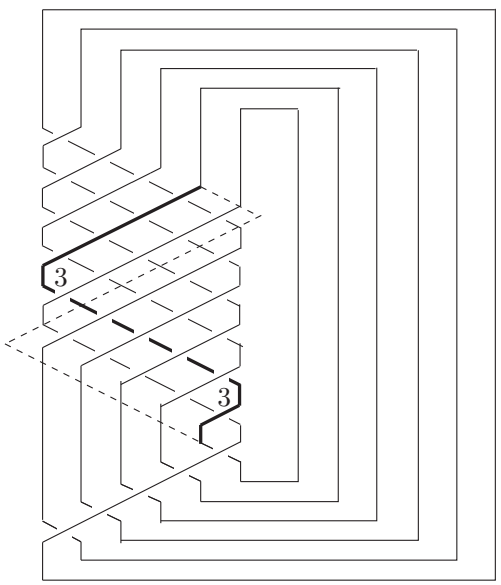

Figure 7. Step 1

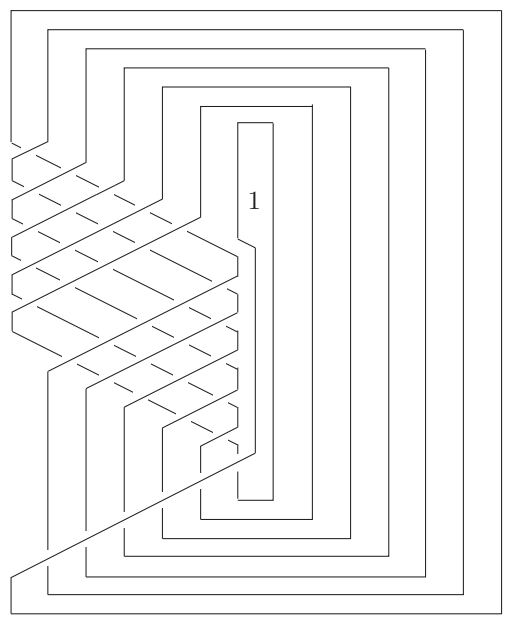

Figure 6. Monogon

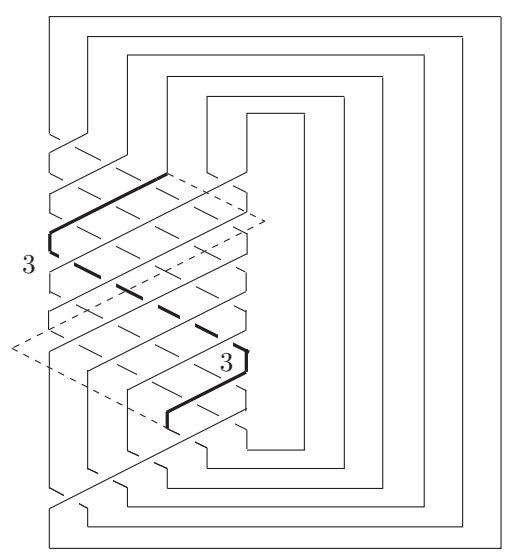

Figure 8. Step 2

When $n \geq 3$, we perform the deformations of $n-2$ steps as in Figures 7 , 8, 9, 10. The deformation of the $k$-th step moves the $(n-k)$-th strand $\gamma_{n-k}$ of the braid except near the last crossing. Any crossing that $\gamma_{n-k}$ passes is made by the $i$-th strand $\gamma_{i}$ and the $j$-th strand $\gamma_{j}$ with $1 \leq i \leq n-k-1$ and $n-k+1 \leq j \leq n$. Note that $\gamma_{i} \cup \gamma_{j}$ has precisely one crossing in each of the two regions bounded by the bold line and the broken line. Hence the deformation of the $k$-th step is accomplished by $2 k(n-k-1)$ RIII moves.

Thus we obtain the desired knot diagram $D(n, n+1)$ as in Figure 11, where the crossings within the triangle can be moved to the bottom of the braid by an isotopy as in Figures 11 and 12 ,

The above deformation is composed of a single RI move, and $(n-1) n / 2+$ $\sum_{k=1}^{n-2} 2 k(n-k-1)=(n-1) n(2 n-1) / 6$ RIII moves. Thus the former half of Theorem 1.1 follows. 


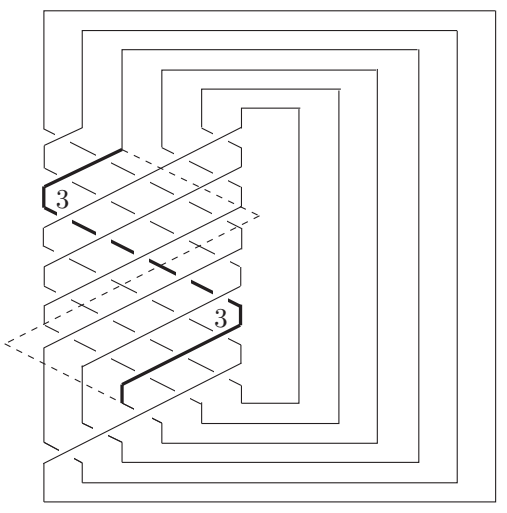

Figure 9. Step 3

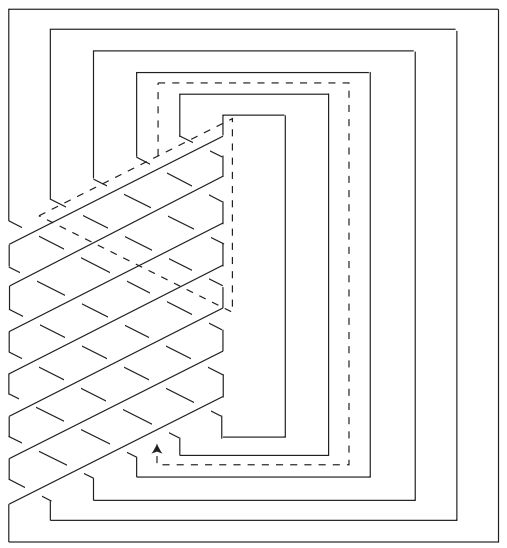

Figure 11. $D(6,7)$

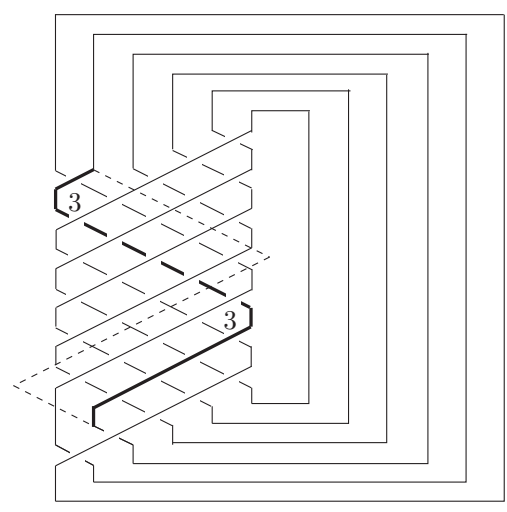

Figure 10. Step 4

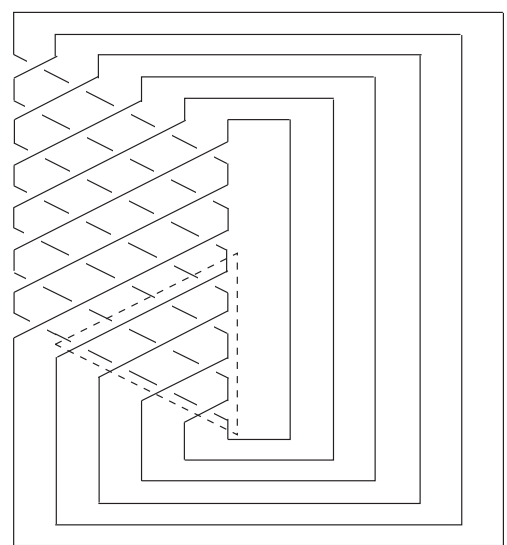

FIGURE 12. $D(6,7)$

\section{Calculation of cowrithe}

In this section, we calculate the cowrithes $x(D(n+1, n))$ and $x(D(n, n+1))$ of the diagrams $D(n+1, n)$ and $D(n, n+1)$.

Lemma 3.1. $x(D(n+1, n))=(n-1) n^{2}(n+4) / 6$ and $x(D(n, n+1))=$ $(n-1) n(n+1)^{2} / 6$.

Before beginning calculations, let us recall the definitions of a chord diagram and a cowrithe.

Let $D$ be a diagram in $S^{2}$ or $\mathbb{R}^{2}$ of a knot $K$. There are an embedding $f: S^{1} \rightarrow S^{3}$ with $f\left(S^{1}\right)=K$ and a projection $\pi: S^{3}-\left\{p_{+}, p_{-}\right\} \cong S^{2} \times(-1,1) \rightarrow S^{2} \times\{0\} \subset S^{3}$ or $\pi: S^{3}-\{\infty\} \cong \mathbb{R}^{2} \times \mathbb{R} \rightarrow \mathbb{R}^{2} \times\{0\} \subset S^{3}$ with $\pi(K)=D$ where $p_{+}, p_{-}$and $\infty$ are points in $S^{3}$ disjoint from $K$. For every crossing $X$, let $X_{1}$ and $X_{2}$ be the preimage points of $X$; that is, $\pi\left(f\left(X_{i}\right)\right)=X$ for $i=1$ and 2. These two points $X_{1}$ and $X_{2}$ are contained in $S^{1}$, and $S^{1}$ is the unit circle in $\mathbb{R}^{2}$. The straight line segment connecting $X_{1}$ and $X_{2}$ in $\mathbb{R}^{2}$ is called the chord for the crossing $X$ and is denoted by $\mathrm{Ch}_{X}$. The chord diagram $\mathrm{Ch}(D)$ of $D$ is the union of $S^{1}$ and the chords 

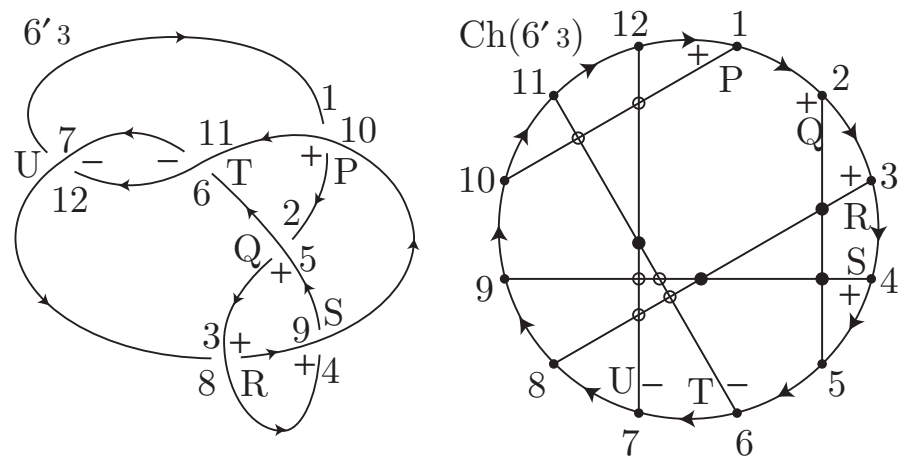

Figure 13. Definition of cowrithe

for all the crossings of $D$. For example, a knot diagram $6_{3}^{\prime}$ and its chord diagram are described in Figure 13 .

We say that two crossings of $D$ are interleaved if the chords for them intersect in a single point. A pair of crossings are interleaved if and only if their preimage points appear alternately on $S^{1}$. We give $D$ an arbitrary orientation. For every pair of interleaved crossings $P$ and $Q$ of $D$, we define the sign of the pair to be the product $(\operatorname{sign} P) \cdot(\operatorname{sign} Q)$. The cowrithe $x(D)$ of $D$ is the sum of signs of all the interleaved pairs of crossings of $D$. Reversing the orientation of $D$ does not change $x(D)$ since it does not change the sign of any crossing. An example of a calculation of cowrithe is described in the right of Figure 13. where we put a black dot at the intersection point of the chords $\mathrm{Ch}_{U}$ and $\mathrm{Ch}_{T}$ to indicate the positive sign of the pair of minus crosssings $U$ and $T$. The white dot at the intersection of the chords $\mathrm{Ch}_{P}$ and $\mathrm{Ch}_{U}$ stands for the negative sign of the pair $P$ and $U$. We can easily see that $x\left(6_{3}^{\prime}\right)=4-6=-2$.
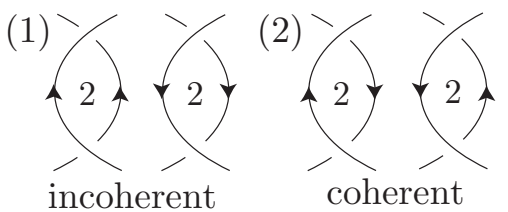

FIGURE 14. Bigon faces

Cowrithe is not a knot invariant. However, it is almost an invariant in the sense that any Reidemeister move changes it at most by a constant.

Theorem $3.2([5])$. An RI move does not change $x(D)$. An RII move deleting a bigon face $f$ increases $x(D)$ by 1 if the orientations of the edges of $f$ are incoherent on the boundary circle of $f$ with respect to an orientation of $D$ as in Figure 14 (1). Otherwise, it does not change $x(D)$. An RIII move changes $x(D)$ by \pm 1 .

Corollary 3.3. Let $D_{1}$ and $D_{2}$ be knot diagrams which represent the same knot. Then, any sequence of Reidemeister moves deforming $D_{1}$ to $D_{2}$ contains at least $\left|x\left(D_{1}\right)-x\left(D_{2}\right)\right|$ RII and RIII moves. 


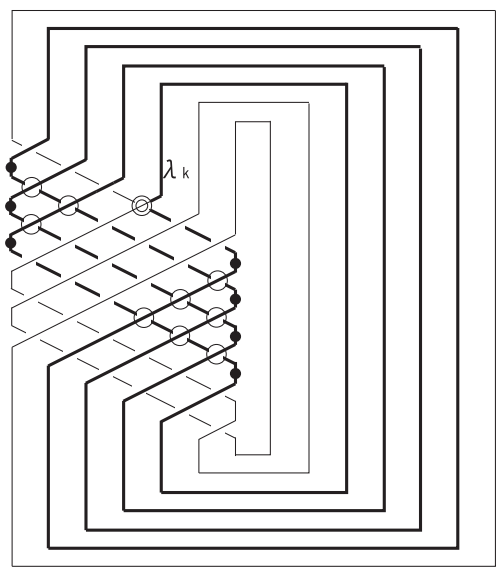

FiguRE 15. $D(7,6)$

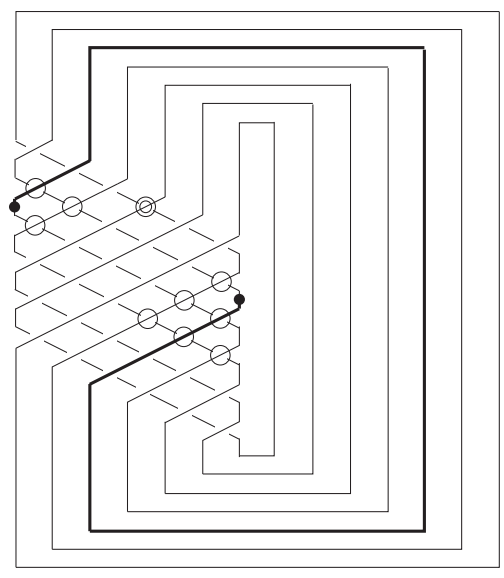

FiguRe 16. Overpath

Proof of Lemma 3.1. First, we calculate $x(D(n+1, n))$.

Since the diagram $D(n+1, n)$ has only positive crossings, its cowrithe is equal to the number of pairs of interleaved crossings of $D$.

The preimage points of the first crossing point corresponding to $b_{k}(1 \leq k \leq n)$ divide the knot $K$ into two arcs. See Figure 15, where $b_{k}$ is the first $b_{4}$ doubly circled. One of the arcs, say $\lambda_{k}$, begins at the undercrossing point of the first $b_{k}$ and goes down along the first strand of the braid, then goes through the second, the third, $\ldots$, the $k$-th strand and then goes back to the first $b_{k}$ at the overcrossing point. (The $i$-th strand is the strand of the braid, which contains the $i$-th points from the left at the top of the braid.) We show that the arc $\lambda_{k}$ contains $2 k(n-1)$ preimage points of crossings other than the endpoints. We take a point between every adjacent pair of an overcrossing point and an undercrossing point on $\lambda_{k}$. Then these points divide $\lambda_{k}$ into $k$ overpaths and $k$ underpaths. An overpath contains no undercrossing points, and an underpath contains no overcrossing points. An overpath is drawn in a bold line in Figure 16. Among these $2 k$ paths, two are incident to the first $b_{k}$ doubly circled and each of them contains $n-k$ preimage points of crossings. Each of the other $2(k-1)$ paths contains $n$ preimage points. Hence the arc $\lambda_{k}$ contains $2(n-k)+2(k-1) n=2 k(n-1)$ preimage points.

There are crossing points for which both preimage points are on $\lambda_{k}$. See Figure 15, where such crossings are circled. $k-2 b_{1}$ 's (the second through the $(k-1)$-st), $k-3 b_{2}$ 's (the second through the $(k-2)$-nd), $\cdots$ and one $b_{k-2}$ (the second), one $b_{n-k+2}$ (the $k$-th), two $b_{n-k+3}$ 's (the $(k-1)$-st and the $k$-th), $\cdots$ and $(k-1) b_{n}$ 's (the second through $k$-th). Each of these $(k-2)(k-1) / 2+(k-1) k / 2=(k-1)^{2}$ crossings and the first $b_{k}$ are not interleaved. Hence the first $b_{k}$ contributes to $x(D(n+1, n))$ by $2 k(n-1)-2(k-1)^{2}$. Any crossing $b_{k}$ has the same contribution by symmetry of the diagram $D(n+1, n)$.

The diagram $D(n+1, n)$ has $n$ crossings corresponding to $b_{k}$ with $1 \leq k \leq n$. We counted contributions doubly. Hence

$$
\begin{gathered}
x(D(n+1, n))=(1 / 2) \sum_{k=1}^{n}\left\{\left(2 k(n-1)-2(k-1)^{2}\right) \times n\right\} \\
=\{(n-1) n(n+1) / 2-(1 / 6)(n-1) n(2(n-1)+1)\} \times n=(n-1) n^{2}(n+4) / 6 .
\end{gathered}
$$




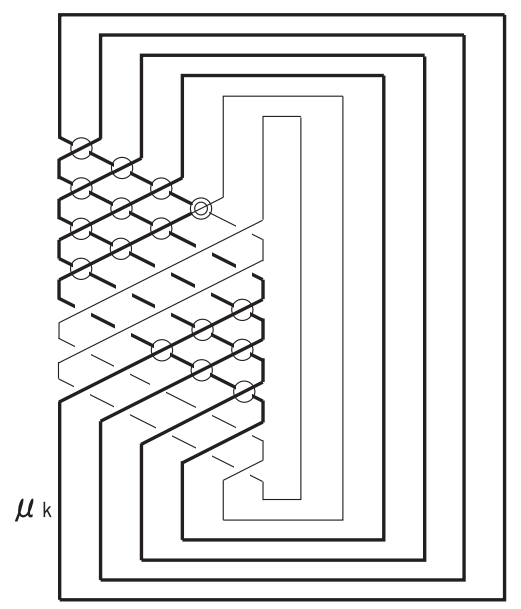

Figure 17. $D(6,7)$

Next, we calculate $x(D(n, n+1))$. The preimage points of the first crossing point corresponding to $b_{k}(1 \leq k \leq n-1)$ divide the knot $K$ into two arcs. See Figure 17, where $b_{k}$ is the first $b_{4}$ doubly circled. One of the arcs, say $\mu_{k}$, begins at the undercrossing point of the first $b_{k}$ and goes up along the first strand of the braid, then goes through the second, the third, ..., the $(k+1)$-st strand, to go back to the first $b_{k}$ at the overcrossing point. The arc $\mu_{k}$ contains $2(k n-1)$ preimage points of crossings other than the endpoints. (Since $\mu_{k}$ consists of $2(k+1$ ) overpaths and underpaths, each of the two paths incident to the first $b_{k}$ contains $k-1$ preimage points and each of the other paths contains $n-1$ preimage points, the arc $\mu_{k}$ contains $2(k-1)+2 k(n-1)=2(k n-1)$ preimage points.) There are crossing points for which both preimage points are on $\mu_{k}: k b_{1}$ 's (the first through the $k$-th), $k-1 b_{2}$ 's (the first through the $(k-1)$-st), $\cdots$ and two $b_{k-1}$ (the first and the second), one $b_{n-k+1}$ (the $(k+1)$-st), two $b_{n-k+2}$ 's (the $k$-th and the $(k+1)$-st), $\cdots$ and $(k-1) b_{n-1}$ 's (the third through $(k+1)$-st). Each of these $k(k+1) / 2-1+(k-1) k / 2=k^{2}-1$ crossings and the first $b_{k}$ are not interleaved. See Figure 17, where such crossings are circled. Hence the first $b_{k}$ contributes to $x(D(n, n+1))$ by $2(k n-1)-2\left(k^{2}-1\right)$. Any crossing $b_{k}$ has the same contribution by symmetry of the diagram $D(n, n+1)$.

The diagram $D(n, n+1)$ has $n+1$ crossings corresponding to $b_{k}$ with $1 \leq k \leq$ $n-1$. Hence, we have

$$
\begin{gathered}
x(D(n, n+1))=(1 / 2) \sum_{k=1}^{n-1}\left\{\left(2(k n-1)-2\left(k^{2}-1\right)\right) \times(n+1)\right\} \\
=(n-1) n(n+1)^{2} / 6 .
\end{gathered}
$$

\section{Proof of Theorem 1.1}

The first half of the theorem has been shown in Section 2 ,

We can obtain easily the estimation for the number of RI moves by using writhe. For any knot diagram $D$, the writhe $w(D)$ is the sum of the sign of all the crossings 
of $D$ with respect to an arbitrary orientation of $D$. Writhe is changed by an RI move by one, and is unchanged by an RII or RIII move. Note that $w(D)$ is unchanged if the orientation of $D$ is reversed. Hence, for two knot diagrams $D_{1}$ and $D_{2}$, we need at least $\left|w\left(D_{1}\right)-w\left(D_{2}\right)\right|$ RI moves to deform $D_{1}$ to $D_{2}$. Because $D(n+1, n)$ has $n^{2}$ positive crossings and no negative crossings, we have $w(D(n+1, n))=n^{2}$. Similarly, $w(D(n, n+1))=(n-1)(n+1)=n^{2}-1$, and hence $|w(D(n+1, n))-w(D(n, n+1))|=1$. Thus we need at least one RI move to deform $D(n+1, n)$ to $D(n, n+1)$.

By Lemma 3.1, the difference of cowrithes is $x(D(n+1, n))-x(D(n, n+1))=$ $(n-1) n^{2}(n+4) / 6-(n-1) n(n+1)^{2} / 6=(n-1) n(2 n-1) / 6$. Hence, by Corollary 3.3. we need at least $(n-1) n(2 n-1) / 6$ RII and RIII moves to deform $D(n+1, n)$ to $D(n, n+1)$. This completes the proof of Theorem 1.1

\section{SOME NOTES FOR GENERALIZATION}

It is very easy to see that $x(D(5,2))=12$ and $x(D(2,5))=10$. However, it seems to be impossible to deform $D(5,2)$ to $D(2,5)$ by a sequence of Reidemeister moves containing at most two RII and RIII moves.

It is very beautiful that two positive link diagrams representing the same link are connected by a sequence of Reidemeister moves which do not create a negative crossing. Note that such a sequence does not contain an RII move. However, the flype operation as shown in Figure 18 does not seem to be realizable by a sequence of Reidemeister moves without an RII move.

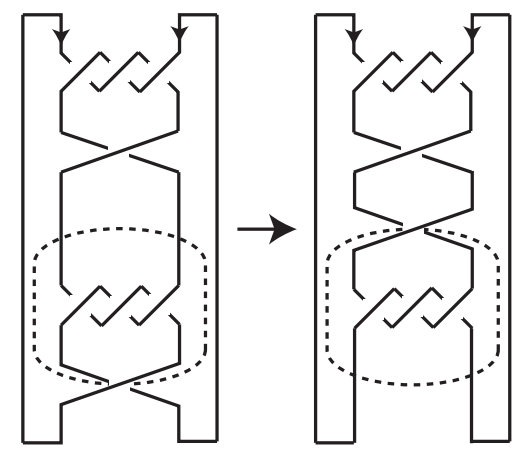

Figure 18. Flyping operation

\section{REFERENCES}

1. V.I. Arnold, Plane curves, their invariants, perestroikas and classifications. Singularities and Bifurcations (V.I. Arnold, ed.) Adv. Sov. Math., 21, Amer. Math. Soc., 1994, 39-91. MR.1310595 (95m:57009)

2. J.W. Alexander and G.B. Briggs, On types of knotted curves. Ann. of Math. (2) 28 (1926/27), 562-586. MR 1502807

3. J. Carter, M. Elhamdadi, M. Saito and S. Satoh, A lower bound for the number of Reidemeister moves of type III. Topology Appl. 153 (2006), 2788-2794. MR2248382 (2007d:57009)

4. T.J. Hagge, Every Reidemeister move is needed for each knot type. Proc. Amer. Math. Soc. 134 (2006), 295-301. MR2170571 (2006g:57010)

5. C. Hayashi, A lower bound for the number of Reidemeister moves for unknotting. J. Knot Theory Ramifications 15 (2006), 313-325. MR2217498(2006m:57005) 
6. J. Hass and T. Nowik, Invariants of knot diagrams. Math. Ann. 342 (2008), 125-137. MR2415317 (2010b:57014)

7. J. Hass and T. Nowik, Unknot diagrams requiring a quadratic number of Reidemeister moves to untangle. Discrete Comput. Geom. 44 (2010), 91-95. MR2639820

8. V.O. Manturov, Knot Theory. Chapman \& Hall/CRC Press, 2004. Appendix A. MR 2068425 (2005d:57008)

9. O.-P. Ostlund, Invariants of knot diagrams and relations among Reidemeister moves. J. Knot Theory Ramifications 10 (2001), 1215-1227. MR1871226 (2002j:57021)

10. K. Reidemeister, Elementare Beründang der Knotentheorie. Abh. Math. Sem. Univ. Hamburg 5 (1926), 24-32.

Department of Mathematical and Physical Sciences, Faculty of Science, Japan Women's University, 2-8-1 Mejirodai, Bunkyo-Ku, Tokyo, 112-8681, JaPAn

E-mail address: hayashic@fc.jwu.ac.jp

Department of Mathematical and Physical Sciences, Faculty of Science, Japan Women's University, 2-8-1 Mejirodai, Bunkyo-Ku, Tokyo, 112-8681, Japan

E-mail address: miwakura@fc.jwu.ac.jp 